hep-th/0411006

KIAS-P04042

\title{
Type 0A matrix model of black hole, integrability and holography
}

\author{
Jaemo Park ${ }^{a *}$ and Takao Suyama ${ }^{b \dagger}$ \\ ${ }^{a}$ Department of Physics, POSTECH, Pohang 790-784, Korea \\ ${ }^{b}$ School of Physics, Korea Institute for Advanced Study, Seoul 130-722, Korea
}

\begin{abstract}
We investigate a deformed matrix model of type 0A theory related to supersymmetric Witten's black hole in two-dimensions, generalization of bosonic model suggested by Kazakov et. al. We find a free field realization of the partition function of the matrix model, which includes Ramond-Ramond perturbations in the type 0A theory. In a simple case, the partition function is factorized into two determinants, which are given by $\tau$ function of an integrable system. We work out the genus expansion of the partition function. Holographic relation with the supersymmetric Witten's black hole is checked by Wilson line computation. Corresponding partition function of the matrix model exhibits a singular behavior, which is interpreted as the point of enhanced $\mathcal{N}=2$ worldsheet supersymmetry. Interesting relation of the deformed matrix model and topological string on a $Z_{2}$ orbifold of conifold is found.
\end{abstract}

\footnotetext{
*jaemo@physics.postech.ac.kr

${ }^{\dagger}$ suyama@kias.re.kr
} 


\section{Introduction}

Kazakov, Kutasov and Kostov [1] propose a matrix model dual to the Sine-Liouville theory, which in turn is conjectured to be equivalent to the 2-D string theory admitting black hole background described by a coset model of $S L(2, R) / U(1)$ type. This is known as the Witten's 2D black hole 2]. Sine-Liouville theory is the deformation of the usual Liouville theory by winding modes or the condensation of vortices. In the matrix model side, winding mode perturbations are described as the insertion of exponentiated Wilson line operator. In [3], it is shown that the duality relation between the 2-D string theory on the Witten's black hole and the deformed matrix model could be understood in terms of gauge theory/gravity theory correspondence. In [3], the proposed matrix model of Kazakov, Kostov and Kutasov [1] was shown to be a gauge theory. This reparaphrased holography was checked by computing the Wilson line expectation value and comparing it with the bulk computation. This check of the holographic relation was made in the context of the bosonic string.

One motivation of this paper is to understand the holographic relation in the context of the type 0A string. Obviously one can consider the Witten's black hole with $\mathcal{N}=1$ worldsheet supersymmetry in the type $0 \mathrm{~A}$ setup. In addition, if we consider the 2-D supergravity action describing low energy theory of the type 0A string theory, we can have a classical 2-D extremal black hole solution 4]. This is argued to be dual to the type $0 \mathrm{~A}$ matrix model with $\mu=0$ and nonzero $f$, where $\mu$ denotes the usual Fermi sea level and $f$ is related to the RamondRamond(RR) flux in the type 0A string theory [5]. Also it is conjectured that non-extremal black holes are described by the Wilson line deformation of the type 0A matrix model [5]. Thus it might be interesting to work out the corresponding matrix models or gauge theories holographically dual to these black hole solutions of the type 0A string theory. In [1], the partition function of the underlying matrix model describing $S L(2, R) / U(1)$ black hole background is given by the $\tau$ function of an integrable system. We might guess that similar integrable structures underlie on the type 0A matrix model dual to 2-D black hole backgrounds. We consider the type 0A matrix model with exponentiated Wilson line operators which correspond to two kinds of winding mode perturbations in the type 0A Liouville theory. Indeed we find a free field realization of the partition function of the type $0 \mathrm{~A}$ matrix model and find that turning on RR flux in the string theory corresponds to the nonzero relative momentum in the free field representation. However the analogue of the Hirota bilinear equation, which would give the needed differential equation governing the partition function, is more complicated than the bosonic case.

The situation is greatly simplified if we consider turning on no RR flux and turning on the same perturbations for both winding modes. This is again related to the Witten's black hole solution with $\mathcal{N}=1$ worldsheet supersymmetry. In this case the partition function is decomposed into two determinants, which are described by the $\tau$ function again. The resulting 
partition function shows singular behavior at two points of the parameter spaces, which indicates the vanishing of the mass gap or the emergence of the new degrees of freedom in the infrared. One of the singularity is well known. The analogous situation in the bosonic case is related to the conifold singularity of a Calabi-Yau space. Using the relation between $c=1$ matrix model at the self-dual radius and topological string on the conifold $[6$, this corresponds to vanishing of the 3-cycle of the conifold and black hole state of the string theory on the conifold becomes massless [7. From the conformal field theory side, this means that the mass gap of the conformal field theory $(\mathrm{CFT})$ vanishes [8]. The other singularity is not known so far. The singularity structure is important since the holographic relation holds for this point of the parameter space, as indicated by the Wilson line calculations. We suggest that this singularity is related to the enhancement of the worldsheet supersymmetry of $\mathcal{N}=1 S L(2, R) / U(1)$ theory to $\mathcal{N}=2$ worldsheet supersymmetry.

Another interesting point is that the compactified radius of the type $0 \mathrm{~A}$ matrix model indicates that the theory is equivalent to some topological string, which is identified with the IIB topological string on a $Z_{2}$ orbifold of the conifold. It is well known that $c=1$ matrix model at the self-dual radius is equivalent to the IIB topological string theory on the conifold $[\underline{6}$. Here we see that type $0 \mathrm{~A}$ version of this relation is going to be useful in understanding the holographic relation. According to [9], the underlying integrable structure of $c=1$ matrix model can be encoded into large symmetries of the conifold. This approach would be useful in elucidating the integrable structures of the type $0 \mathrm{~A}$ matrix models of our interest. Related issues are also discussed in 10

The content of the paper is as follows. In section 2, we briefly review the various equivalences between two-dimensional CFT describing Witten's black hole, Sine-Liouville theory and the corresponding matrix model for bosonic case and introduces some preliminaries needed for type $0 \mathrm{~A}$ generalizations. In section 3 , we introduce a deformed matrix model related to $\mathcal{N}=12-\mathrm{D}$ black hole. In section 4 , we work out the determinant representation and the factorization of the partition function in a simple case. In section 5, we explicitly work out the free energy of the type $0 \mathrm{~A}$ matrix model. In section 6 ,we carry out Wilson line computation and in section 7 , we comment on the relation to topological string. In appendices, determinant representation and free field representation of the partition function are worked out. 


\section{Review of bosonic case and preliminaries for type $0 \mathrm{~A}$ case}

Witten's two-dimensional black hole is described by $S L(2, R) / U(1)$ coset conformal field theory with level $k=\frac{9}{4}[2$. Its two-dimensional geometry is given by

$$
\begin{aligned}
d s^{2} & =\alpha^{\prime} k\left(d r^{2}+\tanh ^{2} r d \theta^{2}\right) \\
\Phi & =\Phi_{0}-\frac{1}{2} \log (\cosh 2 r+1)
\end{aligned}
$$

where $\Phi$ is the usual dilaton field. This is derived by considering the bare Lagrangian of gauged WZW model of $S L(2, R) / U(1)$. For bosonic case there are additional $\alpha^{\prime}$ corrections 11 [12]. According to the conjectures made by Fateev,Zamolodchikov and Zamolodchikov [13], this coset CFT is equivalent to the Sine-Liouville theory

$$
S=\frac{1}{4 \pi} \int d^{2} \sigma\left[(\partial x)^{2}+(\partial \phi)^{2}-Q \hat{R} \phi+\lambda e^{b \phi} \cos R\left(x_{L}-x_{R}\right)\right]
$$

with $R=\frac{3}{2}, Q=2$ and $b=-\frac{1}{2}$. In the paper [1], the matrix model equivalent to the SineLiouville theory is constructed. We consider the $c=1$ matrix quantum mechanics compactified on a circle with radius $R$ with a twisted boundary condition, whose partition function is given by

$$
Z_{N}(\Omega)=\int_{M(2 \pi R)=\Omega^{\dagger} M(0) \Omega} \mathcal{D} M(x) \exp \left(-\operatorname{Tr} \int_{0}^{2 \pi R} d x\left[\frac{1}{2}\left(\partial_{x} M\right)^{2}+V(M)\right]\right),
$$

where $M(x)$ is an $N \times N$ matrix-valued field on the circle and $\Omega$ is a unitary matrix. The form of $V(M)$ is

$$
V(M)=\frac{1}{2} M^{2}-\frac{g}{3 \sqrt{N}} M^{3}
$$

but the precise form of the potential is not important in the double scaling limit. The proposed matrix model is defined by the partition function

$$
Z_{N}(\lambda)=\int \mathcal{D} \Omega e^{\lambda T r\left(\Omega+\Omega^{\dagger}\right)} Z_{N}(\Omega)
$$

In [1], it is shown that the matrix model (2.5) is equivalent to a modified version of the SineLiouville theory

$$
S=\frac{1}{4 \pi} \int d^{2} \sigma\left[(\partial x)^{2}+(\partial \phi)^{2}-2 \hat{R} \phi+\mu e^{-2 \phi}+\lambda e^{(R-2) \phi} \cos R\left(x_{L}-x_{R}\right)\right] .
$$

In a suitable limit where the term $\mu e^{-2 \phi}$ is negligible, the modified theory reduces to the SineLiouville theory, which is equivalent to the black hole CFT.

We consider the generalization to the theories with $\mathcal{N}=1$ worldsheet supersymmetry. Type 0A Sine-Liouville theory contains two worldsheet superfield $X$ with central charge $c=\frac{3}{2}$ and $\phi$ with $c=\frac{3}{2}+6 Q^{2}$. The total central charge $c=15$ determines $Q=\sqrt{2}$. On the other hand, 
$\mathcal{N}=1 S L(2, R) / U(1)$ CFT with level $k$ has the total central charge $c=\frac{3(k+2)}{k}$. From $c=15$ we obtain $k=\frac{1}{Q^{2}}=\frac{1}{2}$. The two-dimensional geometry (2.1) is not renormalized with $\mathcal{N}=1$ worldsheet supersymmetry [14]. Hence the compactification radius $R=\sqrt{\alpha^{\prime} k}$ and we obtain $R=\frac{1}{2}$ with the convention $\alpha^{\prime}=\frac{1}{2}$ and this is the same compactification radius of the time direction $X$ for the matrix model if the holographic relation holds. When we compactify $X$ with the radius $R$, we can have the superaffine theory as well as the usual type $0 \mathrm{~A}$ theory. These two theories differ by the GSO projections [15]. While the superaffine theory correlates the sum over spin structures with the momentum and winding of $X$, the type $0 \mathrm{~A}$ theory does not. For the supercoset model, it is natural to consider the GSO projections which do not correlate the fermionic projections with the momentum and winding modes, which we call diagonal GSO projections. Thus we look for the holographic relation between the type $0 \mathrm{~A}$ theory and $\mathcal{N}=1$ supercoset with the diagonal GSO projections. For the type 0A case, matrix model should be suitably generalized and this will be explained in the next section.

\section{$3 \quad$ Matrix model of type 0A string}

It is proposed in [15] that type 0A string in two dimensions has a matrix model realization which is a quantum mechanics of a complex matrix. Our interest is on type 0A string whose "time direction", i.e. the direction corresponding to the free boson, is compactified on $S^{1}$. The compactification is realized simply by letting the time direction of the quantum mechanical system be periodic. As mentioned in [15], the background RR flux can be introduced by adding a Chern-Simons like term. The action of the matrix model is

$$
S=\int_{0}^{2 \pi R} d x \operatorname{Tr}\left[\left|D_{x} t\right|^{2}-\frac{1}{2 \alpha^{\prime}}|t|^{2}+i f(A-\tilde{A})\right] .
$$

Here $t(x)$ is an $N \times N$ complex matrix field on $S^{1}$ with radius $R . t(x)$ couples to the $U(N)$ gauge fields $A, \tilde{A}$ through the covariant derivative

$$
D_{x} t=\partial_{x} t-i A t+i t \tilde{A}
$$

The constant $f$ is the strength of the RR flux. If we interpret the matrix model as a quantum mechanics based on D0-branes [17, the theory describes $f$ D0-branes in type 0A theory

Note that there is $i$ in front of the Chern-Simons like term since we have done the Wick rotation. ¿From now on, we set $\alpha^{\prime}=1 / 2$.

We fix the gauge by the condition $\partial_{x} A=\partial_{x} \tilde{A}=0$. Then the remaining degrees of freedom of gauge fields are the zero modes $A_{0}, \tilde{A}_{0}$. These zero modes can be absorbed by defining a new 
field $\tilde{t}(x)$ as

$$
\tilde{t}(x)=e^{-i A_{0} x} t(x) e^{i \tilde{A}_{0} x} .
$$

The new field $\tilde{t}(x)$ obeys a twisted boundary condition

$$
\tilde{t}(x+2 \pi R)=U \tilde{t}(x) V^{\dagger}
$$

where

$$
U=\exp \left(-2 \pi i R A_{0}\right), \quad V=\exp \left(-2 \pi i R \tilde{A}_{0}\right)
$$

The action for $\tilde{t}$ is

$$
S=\int_{0}^{2 \pi R} d x \operatorname{Tr}\left[\left|\partial_{x} \tilde{t}\right|^{2}-|\tilde{t}|^{2}\right]-f \operatorname{Tr}(\log U-\log V),
$$

and the partition function is

$$
Z_{N}=\int \mathcal{D} U \mathcal{D} V \mathcal{D} \tilde{t}(x) e^{-S}
$$

where $\mathcal{D} U, \mathcal{D} V$ are the Haar measures on $U(N)$.

One can perturb the action (3.6) by the Wilson line operators. For the gauge choice we chose, the Wilson line operators for the gauge fields $A, \tilde{A}$ are proportional to $\operatorname{Tr} U, \operatorname{Tr} V$, respectively. We will consider an action with a general perturbation

$$
Z_{N}(\lambda, \tilde{\lambda})=\int \mathcal{D} U \mathcal{D} V \mathcal{D} \tilde{t}(x) e^{-S+W(U)+\tilde{W}(V)},
$$

where

$$
W(U)=\sum_{n \neq 0} \lambda_{n} \operatorname{Tr} U^{n}, \quad \tilde{W}(V)=\sum_{n \neq 0} \tilde{\lambda}_{n} \operatorname{Tr} V^{n} .
$$

Note that the above path integral is not well-defined since the potential is not bounded from below. In the following, we will evaluate the path integral by first replacing the unbounded potential with the ordinary harmonic potential with frequency $\omega$, and then continuing $\omega$ to $i$.

The path integral of $\tilde{t}(x)$ can be performed explicitly, since the system consists of $N^{2}$ harmonic oscillators with the twisted boundary conditions

$$
\tilde{t}_{i j}(x+2 \pi R)=z_{i} \tilde{z}_{j}^{-1} \tilde{t}_{i j}(x)
$$

where $z_{i}, \tilde{z}_{i}$ are eigenvalues of $U, V$, respectively. The partition function is, after integration,

$$
\begin{aligned}
Z_{N}(\lambda, \tilde{\lambda})= & \frac{1}{(N !)^{2}} \oint \prod_{i} \frac{d z_{i}}{2 \pi i z_{i}} \frac{d \tilde{z}_{i}}{2 \pi i \tilde{z}_{i}} e^{w\left(z_{i}\right)+\tilde{w}\left(\tilde{z}_{i}\right)} \\
& \times \prod_{i<j}\left|z_{i}-z_{j}\right|^{2}\left|\tilde{z}_{i}-\tilde{z}_{j}\right|^{2} \prod_{i, j} \frac{z_{i} \tilde{z}_{j}}{\left(z_{i} q^{1 / 2}-\tilde{z}_{j} q^{-1 / 2}\right)\left(\tilde{z}_{j} q^{1 / 2}-z_{i} q^{-1 / 2}\right)}
\end{aligned}
$$


where $q=\exp (2 \pi i R)$ and

$$
w(z)=f \log z+\sum_{n \neq 0} \lambda_{n} z^{n}, \quad \tilde{w}(\tilde{z})=-f \log z+\sum_{n \neq 0} \tilde{\lambda}_{n} \tilde{z}^{n}
$$

Comparing with the bosonic case[1], we have two kinds of perturbations instead of one. These correspond to the two winding mode perturbations in the Liouville theory. In the T-dual 0B theory, these correspond to the two kinds of momentum mode perturbations of the Liouville theory and to the deformations of two Fermi seas in the dual matrix quantum mechanics [16], [15]. In later sections, we show some evidences that there is holography between type 0A matrix model with a suitable deformation without RR flux and $\mathcal{N}=1$ Witten's black hole. We expect that more general cases with two kinds of winding mode perturbations correspond to black hole solutions with two parameters in [4].

Note also that turning on RR flux simply corresponds to turning on $\log z$ perturbation. It might be interesting to find integrable structures associated with the backgrounds with RR flux. In the appendix $\mathrm{B}$ we show that the partition function admits a simple free field realization which generalize the result at [19] where the free field realization for the case without RR flux was discussed. There we see that turning on RR flux corresponds to nonzero relative momentum of the two free bosons appearing in the free field realization. In the appendix $\mathbb{B}$ we derive the analogue of the Hirota bilinear differential equation for the model. However the resulting equation is more complicated and we do not reduce the equation into a known form of $\tau$ functions. In the next section we show that the equation could be explicitly evaluated to give the product of the $\tau$ function when two winding modes are the same, which dictates that there should be no RR flux. Unlike the discussion in [19], this is the exact expression including nonperturbative corrections. Thus it might be interesting to work out nonperturbative terms and to compare the expression with the corresponding computations in the Liouville theory as is done in [20].

\section{Determinant representation and factorization}

The expression (3.11) for the partition function can also be rewritten as

$$
\begin{aligned}
Z_{N}(\lambda, \tilde{\lambda}) & =\frac{1}{(N !)^{2}} \oint \prod_{i=1}^{N} \frac{d z_{i}}{2 \pi i} \frac{d \tilde{z}_{i}}{2 \pi i} e^{w\left(z_{i}\right)+\tilde{w}\left(\tilde{z}_{i}\right)} \operatorname{det}_{i j}\left(\frac{1}{z_{i} q^{1 / 2}-\tilde{z}_{j} q^{-1 / 2}}\right) \operatorname{det}_{i j}\left(\frac{1}{\tilde{z}_{j} q^{1 / 2}-z_{i} q^{-1 / 2}}\right) \\
& =\frac{1}{(N !)^{2}} \oint \prod_{i=1}^{N} \frac{d z_{i}}{2 \pi i} \frac{d \tilde{z}_{i}}{2 \pi i} \operatorname{det}_{i j}\left(\frac{e^{\left(w\left(z_{i}\right)+\tilde{w}\left(\tilde{z}_{j}\right)\right) / 2}}{z_{i} q^{1 / 2}-\tilde{z}_{j} q^{-1 / 2}}\right) \operatorname{det}_{i j}\left(\frac{e^{\left(\tilde{w}\left(\tilde{z}_{j}\right)+w\left(z_{i}\right)\right) / 2}}{\tilde{z}_{j} q^{1 / 2}-z_{i} q^{-1 / 2}}\right),
\end{aligned}
$$


by using the identity

$$
\frac{\Delta(a) \Delta(b)}{\prod_{i j}\left(a_{i}-b_{j}\right)}=\operatorname{det}_{i j}\left(\frac{1}{a_{i}-b_{j}}\right),
$$

where $\Delta(a)$ is the Vandermonde determinant.

As in the bosonic case, it is more convenient to consider the grand canonical partition function $Z(\lambda, \tilde{\lambda} ; \mu)$ defined as follows,

$$
Z(\lambda, \tilde{\lambda} ; \mu)=\sum_{N=0}^{\infty} e^{2 \pi R \mu N} Z_{N}(\lambda, \tilde{\lambda}) .
$$

This partition function has the following determinant representation

$$
Z(\lambda, \tilde{\lambda} ; \mu)=\operatorname{Det}\left(1+e^{2 \pi R \mu} \mathcal{K}\right)
$$

where

$$
\begin{aligned}
\mathcal{K}\left(z, z^{\prime}\right) & =\oint \frac{d z^{\prime \prime}}{2 \pi i} K\left(z, z^{\prime \prime}\right) \tilde{K}\left(z^{\prime \prime}, z^{\prime}\right), \\
K\left(z, z^{\prime}\right) & =\frac{e^{\left(w(z)+\tilde{w}\left(z^{\prime}\right)\right) / 2}}{z q^{1 / 2}-z^{\prime} q^{-1 / 2}}, \\
\tilde{K}\left(z, z^{\prime}\right) & =\frac{e^{\left(\tilde{w}(z)+w\left(z^{\prime}\right)\right) / 2}}{z q^{1 / 2}-z^{\prime} q^{-1 / 2}} .
\end{aligned}
$$

The calculation is summarized in appendix A

When $K=\tilde{K}$, the determinant is factorized into two factors,

$$
Z(\lambda, \tilde{\lambda} ; \mu)=\operatorname{Det}\left(1+i e^{\pi R \mu} K\right) \cdot \operatorname{Det}\left(1-i e^{\pi R \mu} \tilde{K}\right)
$$

Note that in this case the RR flux $f$ must vanish and $\lambda_{n}=\tilde{\lambda}_{n}$. Remarkably, each determinant factor is the partition function investigated in [1] with the chemical potential replaced with $(\mu \pm i / 2 R) / 2$. Therefore, $Z(\lambda, \tilde{\lambda} ; \mu)$ can be obtained by applying the same technique used in [1.

When all $\lambda, \tilde{\lambda}$ 's vanish, the determinants can be calculated explicitly. One can show that ${ }^{3}$

$$
\begin{aligned}
K \cdot z^{n} & =\oint \frac{d z^{\prime}}{2 \pi i} K\left(z, z^{\prime}\right)\left(z^{\prime}\right)^{n} \\
& = \begin{cases}0, & (n \geq f / 2), \\
q^{n+1 / 2-f / 2} z^{n}, & (n<f / 2),\end{cases} \\
\tilde{K} \cdot z^{n} & =\oint \frac{d z^{\prime}}{2 \pi i} \tilde{K}\left(z, z^{\prime}\right)\left(z^{\prime}\right)^{n} \\
& = \begin{cases}0, & (n \geq-f / 2), \\
q^{n+1 / 2+f / 2} z^{n}, & (n<-f / 2),\end{cases}
\end{aligned}
$$

\footnotetext{
${ }^{3}$ These calculations are done with $q=\exp (2 \pi \omega R)>1$ and $\omega$ is analytically continued after the contour integrations.
} 


$$
\begin{aligned}
\mathcal{K} \cdot z^{n} & =\oint \frac{d z^{\prime}}{2 \pi i} \mathcal{K}\left(z, z^{\prime}\right)\left(z^{\prime}\right)^{n} \\
& = \begin{cases}0, & (n \geq-|f| / 2), \\
q^{2 n+1} z^{n}, & (n<-|f| / 2) .\end{cases}
\end{aligned}
$$

We have assumed that $f / 2$ is an integer. Therefore one obtains

$$
\begin{aligned}
\operatorname{Det}\left(1+e^{2 \pi R \mu} \mathcal{K}\right) & =\prod_{n<-|f| / 2}\left(1+i e^{\pi R \mu} q^{n+1 / 2}\right)\left(1-i e^{\pi R \mu} q^{n+1 / 2}\right) \\
& \neq \operatorname{Det}\left(1+i e^{\pi R \mu} K\right) \cdot \operatorname{Det}\left(1-i e^{\pi R \mu} \tilde{K}\right) .
\end{aligned}
$$

This implies that the grand canonical partition function is not factorized into two determinants in a similar way as in (4.8) when the RR flux is turned on.

\section{$5 \quad$ Free energy of $0 \mathrm{~A}$ matrix model with perturbation}

\subsection{Review of bosonic case}

In [1] the $\mathrm{c}=1$ matrix model with a perturbation is investigated and its free energy is calculated perturbatively. The grand canonical partition function of this system is defined similarly to (4.3), and this partition function also has the following determinant representation,

$$
Z(\lambda, \mu)=\operatorname{Det}\left(1+e^{2 \pi R \mu} K\right)
$$

where

$$
K\left(z, z^{\prime}\right)=\frac{e^{u(z)+u\left(z^{\prime}\right)}}{z q^{1 / 2}-z^{\prime} q^{-1 / 2}}, \quad u(z)=\frac{1}{2} \sum_{n \neq 0} \lambda_{n} z^{n} .
$$

It is shown in [1] that when all $\lambda$ 's except for $\lambda_{ \pm 1}$ vanish corresponding to the Sine-Liouville theory, the partition function $Z(\lambda, \mu)$ is obtained by solving a non-linear differential equation. For $\chi(\lambda, \mu)=\partial_{\mu}^{2} F(\lambda, \mu)$, where $F(\lambda, \mu)=\log Z(\lambda, \mu)$, the differential equation is

$$
\frac{1}{4 \lambda} \partial_{\lambda}\left(\lambda \partial_{\lambda} \chi(\lambda, \mu)\right)+\partial_{\mu}^{2} \exp \left[-\left(\frac{\sin \left(\partial_{\mu} / 2\right)}{\partial_{\mu} / 2}\right)^{2} \chi(\lambda, \mu)\right]=0
$$

where $\lambda \propto \sqrt{\lambda_{+1} \lambda_{-1}}$ and

$$
\frac{\sin \left(\partial_{\mu} / 2\right)}{\partial_{\mu} / 2}=\sum_{n=0}^{\infty}(-1)^{n} \frac{2^{-2 n}}{(2 n+1) !} \partial_{\mu}^{2 n}
$$


$F(\lambda=0, \mu)$ can be calculated explicitly,

$$
\begin{aligned}
\partial_{\mu}^{3} F(\lambda=0, \mu) & =\partial_{\mu}^{3} \prod_{n=0}^{\infty} \log \left(1+e^{2 \pi R \mu} q^{-n-1 / 2}\right) \\
& =R \operatorname{Im} \int_{0}^{\infty} d t e^{-i t(\mu-i \epsilon)} \frac{t / 2}{\sinh (t / 2)} \frac{t / 2 R}{\sinh (t / 2 R)},
\end{aligned}
$$

where $\epsilon=+0$, and this quantity can be used as a boundary condition to the solution of the differential equation (5.3).

To solve the equation (5.3), $\chi(\lambda, \mu)$ is assumed to have a perturbative expansion whose expansion parameter is a power of $\lambda$ and the coefficient at each order is a function of $y \propto \frac{\mu}{\lambda^{2 /(2-R)}}$. Substituting this perturbative series into (5.3), the differential equation is reduced to an infinite number of second order differential equations. In [1], they are solved explicitly up to genus one.

\subsection{Type 0 A case}

We would like to evaluate the determinant

$$
Z_{ \pm}(\lambda, \mu)=\left.\operatorname{Det}\left(1 \pm i e^{\pi R \mu} K\right)\right|_{\lambda=\tilde{\lambda}}
$$

from which the grand canonical partition function of type $0 \mathrm{~A}$ matrix model is obtained as

$$
Z(\lambda, \tilde{\lambda}=\lambda ; \mu)=Z_{+}(\lambda, \mu) Z_{-}(\lambda, \mu) .
$$

One can see that $Z_{ \pm}(\lambda, \mu)$ is the same function as the bosonic partition function (5.1) with $\mu$ replaced with $(\mu \pm i / 2 R) / 2$. Therefore, when all $\lambda$ 's except for $\lambda_{ \pm 1}$ are turned off, $Z_{ \pm}(\lambda, \mu)$ can also be determined by solving the differential equation

$$
\frac{1}{4 \lambda} \partial_{\lambda}\left(\lambda \partial_{\lambda} \chi_{ \pm}\left(\lambda, \mu^{\prime}\right)\right)+\partial_{\mu^{\prime}}^{2} \exp \left[-\left(\frac{\sin \left(\partial_{\mu^{\prime}} / 2\right)}{\partial_{\mu^{\prime}} / 2}\right)^{2} \chi_{ \pm}\left(\lambda, \mu^{\prime}\right)\right]=0,
$$

where $\mu^{\prime}=\mu / 2$ and $\chi_{ \pm}(\lambda, \mu)=\partial_{\mu^{\prime}}^{2} \log Z_{ \pm}(\lambda, \mu)$.

$F_{ \pm}(\lambda=0, \mu)$ can be calculated explicitly as follows,

$$
\begin{aligned}
\partial_{\mu^{\prime}}^{3} F_{ \pm}(\lambda=0, \mu)= & \frac{R}{i} \int_{0}^{\infty} d t e^{-i t\left(\mu^{\prime} \pm i / 4 R-i \epsilon\right)} \frac{t / 2}{\sinh (t / 2)} \frac{t / 2 R}{\sinh (t / 2 R)} \\
& -\frac{R}{i} \int_{0}^{\infty} d t e^{+i t\left(\mu^{\prime} \pm i / 4 R+i \epsilon\right)} \frac{t / 2}{\sinh (t / 2)} \frac{t / 2 R}{\sinh (t / 2 R)} \\
= & -2 R \int_{0}^{\infty} d t \sin \left(\left(\mu^{\prime} \pm i / 4 R\right) t\right) \frac{t / 2}{\sinh (t / 2)} \frac{t / 2 R}{\sinh (t / 2 R)} \\
= & 2 R \operatorname{Im} \int_{0}^{\infty} d t e^{-i t\left(\mu^{\prime}-i \epsilon\right)} \frac{t / 2}{\sinh (t / 2)} \frac{t / 4 R}{\sinh (t / 4 R)} \\
& \mp 2 i R \operatorname{Re} \int_{0}^{\infty} d t e^{-i t\left(\mu^{\prime}-i \epsilon\right)} \frac{t / 2}{\sinh (t / 2)} \frac{t / 4 R}{\cosh (t / 4 R)} .
\end{aligned}
$$


¿From this expression, one can obtain the asymptotic expansion of $\chi_{ \pm}(\lambda=0, \mu)$

$$
\begin{aligned}
\chi_{ \pm}(\lambda=0, \mu) \sim & -2 R \log \mu^{\prime}+2 R \sum_{N=1}^{\infty} f_{N}(2 R) \mu^{\prime-2 N} \\
& \mp 2 i R \sum_{N=0}^{\infty} g_{N}(2 R) \mu^{\prime-2 N-1},
\end{aligned}
$$

where

$$
\begin{aligned}
& f_{N}(2 R)=(2 N-1) ! \sum_{k=0}^{N} \frac{\left|2^{2 N-2 k}-2\right|\left|2^{2 k}-2\right|}{(2 N-2 k) !(2 k) !}\left|B_{2 N-2 k} B_{2 k}\right|(2 R)^{-2 k} 2^{-2 N}, \\
& g_{N}(2 R)=(2 N) ! \sum_{k=0}^{N} \frac{\left|2^{2 N-2 k}-2\right|}{(2 N-2 k) !(2 k) !}\left|B_{2 N-2 k} E_{2 k}\right|(2 R)^{-2 k-1} 2^{-2 N-1} .
\end{aligned}
$$

Here $B_{k}, E_{k}$ are the Bernoulli number and the Euler number, respectively,

$$
\begin{aligned}
\frac{x}{e^{x}-1} & =\sum_{k=0}^{\infty} \frac{B_{k}}{k !} x^{k}, \\
\operatorname{sech} x & =\sum_{k=0} \frac{E_{k}}{k !} x^{k} .
\end{aligned}
$$

The asymptotic expansion (5.10) will be used as a boundary condition in solving the differential equation.

We make an ansatz for $\chi_{ \pm}(\lambda, \mu)$ as follows,

$$
\chi_{ \pm}(\lambda, \mu)=-2 R \log \mu^{\prime}+\sum_{n=0}^{\infty} \mu^{\prime-n} \chi_{ \pm, n}(z)
$$

where $\chi_{ \pm, n}(z)$ is a power series in $z=\lambda^{2} / \mu^{\prime 2-2 R}$, following [1], and obeys the boundary condition

$$
\begin{aligned}
\chi_{ \pm, 0}(0) & =0, \\
\chi_{ \pm, 2 n}(0) & =2 R f_{n}(2 R), \\
\chi_{ \pm, 2 n+1}(0) & =\mp 2 i R g_{n}(2 R) .
\end{aligned}
$$

According to [1], we make a change of variables from $\left(\mu^{\prime}, z\right)$ to $(\lambda, y)$ where

$$
y=z^{-1 /(2-2 R)}=\frac{\mu^{\prime}}{\lambda^{1 /(1-R)}} .
$$

Then the result is

$$
\begin{aligned}
\chi_{ \pm}(\lambda, \mu) & =-\frac{2 R}{1-R} \log \lambda+\sum_{n=0}^{\infty} \lambda^{-n /(1-R)} X_{ \pm, n}(y) \\
X_{ \pm, 0}(y) & =-2 R \log y+\chi_{ \pm, 0}\left(y^{-1 /(2-2 R)}\right) \\
X_{ \pm, n}(y) & =y^{-n} \chi_{ \pm, n}\left(y^{-1 /(2-2 R)}\right) . \quad(n \geq 1)
\end{aligned}
$$


Substituting this ansatz into (5.8) and setting to zero the coefficient of each power of $\lambda$, we obtain the following set of equations,

$$
\left(y \partial_{y}+n\right)^{2} X_{ \pm, n}(y)+4(1-R)^{2} \partial_{y}^{2}\left(e^{-X_{ \pm, 0}(y)} p_{n}\left[X_{ \pm}\right]\right)=0
$$

where $p_{n}\left[X_{ \pm}\right]$is defined as follows,

$$
\exp \left[-\sum_{n=0}^{\infty} x^{n}\left(\frac{\sin \left(x \partial_{y} / 2\right)}{x \partial_{y} / 2}\right)^{2} X_{ \pm, n}(y)\right]=\sum_{n=0}^{\infty} x^{n} e^{-X_{ \pm, 0}(y)} p_{n}\left[X_{ \pm}\right] .
$$

Explicit forms of $p_{n}\left[X_{ \pm}\right]$are

$$
\begin{aligned}
p_{0}\left[X_{ \pm}\right]= & 1, \\
p_{1}\left[X_{ \pm}\right]= & -X_{ \pm, 1}, \\
p_{2}\left[X_{ \pm}\right]= & -X_{ \pm, 2}+\frac{1}{12} \partial_{y}^{2} X_{ \pm, 0}+\frac{1}{2}\left(X_{ \pm, 1}\right)^{2} \\
p_{3}\left[X_{ \pm}\right]= & -X_{ \pm, 3}+\frac{1}{12} \partial_{y}^{2} X_{ \pm, 1}-\frac{1}{12} X_{ \pm, 1} \partial_{y}^{2} X_{ \pm, 0}+X_{ \pm, 1} X_{ \pm, 2}-\frac{1}{6}\left(X_{ \pm, 1}\right)^{3}, \\
p_{4}\left[X_{ \pm}\right]= & -X_{ \pm, 4}+\frac{1}{12} \partial_{y}^{2} X_{ \pm, 2}-\frac{1}{360} \partial_{y}^{4} X_{ \pm, 0}-\frac{1}{12} X_{ \pm, 1} \partial_{y}^{2} X_{ \pm, 1}+X_{ \pm, 1} X_{ \pm, 3} \\
& +\frac{1}{288}\left(\partial_{y}^{2} X_{ \pm, 0}\right)^{2}-\frac{1}{12} \partial_{y}^{2} X_{ \pm, 0} X_{ \pm, 2}+\frac{1}{2}\left(X_{ \pm, 2}\right)^{2} \\
& +\frac{1}{24}\left(X_{ \pm, 1}\right)^{2} \partial_{y}^{2} X_{ \pm, 0}-\frac{1}{2}\left(X_{ \pm, 1}\right)^{2} X_{ \pm, 2}+\frac{1}{24}\left(X_{ \pm, 1}\right)^{4}, \\
& \text { etc. }
\end{aligned}
$$

(i) $n=0$

The differential equation which we have to solve is

$$
\left(y \partial_{y}\right)^{2} X_{ \pm, 0}(y)+4(1-R)^{2} \partial_{y}^{2} e^{-X_{ \pm, 0}(y)}=0 .
$$

In [1] this equation is solved and the solution is

$$
y=e^{-X_{ \pm, 0} / 2 R}-(2 R-1) e^{(1-2 R) X_{ \pm, 0} / 2 R},
$$

which is valid for $0<R<1$. For $R>1$, there is no solution satisfying the boundary condition (5.17). When $R=1 / 2$, which would be relevant to the fermionic cigar geometry, the solution has the simple form

$$
X_{ \pm, 0}=-\log y .
$$

Then the tree level contribution to $F_{ \pm}(\lambda, \mu)$ is

$$
\begin{aligned}
\left.F_{ \pm}(\lambda, \mu)\right|_{\text {tree }} & =-\frac{1}{2}\left(\mu^{\prime}\right)^{2} \log \mu^{\prime}+A_{1} \mu^{\prime} \lambda^{2}+A_{2} \lambda^{4} \\
& =-\frac{1}{2}\left(\mu^{\prime}\right)^{2} \log \mu^{\prime}+\mu^{\prime 2}\left(A_{1} z+A_{2} z^{2}\right),
\end{aligned}
$$


where $z=\lambda^{2} / \mu^{\prime}$. We have omitted terms which depend only on $\mu^{\prime}$. The constants $A_{1}, A_{2}$ can be fixed by matching this expression with the (3.7) of [1] with $R=1$, and the result is $A_{1}=-1, A_{2}=0$.

(ii) $n \geq 1$

From now on, we focus on the case $R=1 / 2$.

One can see that $p_{n}\left[X_{ \pm}\right]$has the structure

$$
p_{n}\left[X_{ \pm}\right]=-X_{ \pm, n}+\tilde{p}_{n}\left[X_{ \pm}\right]
$$

where $\tilde{p}_{n}\left[X_{ \pm}\right]$only contains $X_{ \pm, m}$ with $m<n$. Then the equation (5.18) is

$$
y(1-y) \partial_{y}^{2} X_{ \pm, n}+(2-(2 n+1) y) \partial_{y} X_{ \pm, n}-n^{2} X_{ \pm, n}=\partial_{y}^{2}\left(y \tilde{p}_{n}\left[X_{ \pm}\right]\right) .
$$

This equation can be solved order by order in $n$. It is remarkable that the equation LHS $=0$ is the hypergeometric equation, and therefore the general solution would have singularities at $y=0,1$.

The solutions which satisfy the boundary conditions (5.17) are as follows,

$$
\begin{aligned}
& X_{ \pm, 1}=\mp \frac{i}{2 y}, \\
& X_{ \pm, 2}=-\frac{1}{24 y^{2}}+\frac{1}{8(y-1)^{2}}, \\
& X_{ \pm, 3}=\mp i\left(\frac{1}{24 y^{3}}+\frac{1}{6(y-1)^{3}}+\frac{1}{8(y-1)^{4}}\right), \\
& X_{ \pm, 4}=-\frac{7}{320 y^{4}}+\frac{3}{64(y-1)^{4}}+\frac{17}{32(y-1)^{5}}+\frac{5}{8(y-1)^{6}},
\end{aligned}
$$

These results suggest that for general $n$ the solution $X_{ \pm, n}$ would have the following simple form

$$
X_{ \pm, n}=\frac{c}{y^{n}}+\sum_{k=n}^{2 n-2} \frac{c_{k}}{(y-1)^{k}} .
$$

where $c, c_{k}$ are rational numbers.

Now $F_{ \pm}(\lambda, \mu)$ can be obtained by integrating twice, and we obtain with $z=\frac{1}{y}$ when $R=\frac{1}{2}$ and $y=\frac{\mu^{\prime}}{\lambda^{2}}$

$$
\begin{aligned}
& F_{ \pm, 1}(\lambda, \mu)=\mp \frac{i}{2} \mu^{\prime} \log \mu^{\prime}+a_{1}^{ \pm} \mu^{\prime} z \\
& F_{ \pm, 2}(\lambda, \mu)=-\frac{1}{12} \log \mu^{\prime}-\frac{1}{8} \log (1-z) \\
& F_{ \pm, 3}(\lambda, \mu)=\mp \frac{i}{\mu^{\prime}}\left(\frac{1}{48}+\frac{1}{12(1-z)}+\frac{z}{48(1-z)^{2}}\right) \\
& F_{ \pm, 4}(\lambda, \mu)=\frac{1}{\mu^{\prime 2}}\left(-\frac{7}{1920}+\frac{1}{128(1-z)^{2}}+\frac{17 z}{384(1-z)^{3}}+\frac{z^{2}}{32(1-z)^{4}}\right)
\end{aligned}
$$

etc. 
Note that the terms depending only on $\mu^{\prime}$ in $F_{ \pm, 0}, F_{ \pm, 2}$ are a half of the corresponding part of the usual genus expansion of the type 0A matrix model. Also all of $F_{+, 2 n+1}$ terms are cancelled against $F_{-, 2 n+1}$ in the final expression of the free energy.

Note that we have two singularities in the expansion at $y=0$ and $y=1$. The singularities at $y=0$ corresponds to the limit $\mu^{\prime} \rightarrow 0$. This singularity appears even when we turn off the deformations of the type 0A matrix model. For the bosonic case, this is interpreted as the vanishing of the mass gap of the corresponding conformal field theory on the Witten's black hole background and the same interpretation could be applied to the type 0A case. If we use the relation between the $\mathrm{c}=1$ matrix model at the self-dual radius and the topological string on the conifold, this singularity at $y=0$ corresponds to the conifold singularity of the topological string. The vanishing of the mass gap is translated into the appearance of the massless black hole at the conifold point. Thus new states are added to the string perturbation theory near the conifold. The singularities at $y=1$ is hitherto unknown. ¿From the Wilson line computation in the next section, we propose that this corresponds to the point of the enhancement of the worldsheet supersymmetry from $\mathcal{N}=1$ to $\mathcal{N}=2$. It is desirable to collect further evidences for this proposal.

Similar calculations can be done for the bosonic case with $R=1$ and type 0B case with $R=1 / 2$. Type $0 \mathrm{~B}$ case has the same differential equation as the bosonic case but with the different boundary conditions, that is, the free energy of type $0 \mathrm{~B}$ without perturbation is twice of that of bosonic string.

Firstly, consider the bosonic case. The boundary condition at $\lambda=0$ is

$$
\chi_{b}(\lambda=0, \mu) \sim-\log \mu+\sum_{N=1}^{\infty} f_{N}(1) \mu^{-2 N} .
$$

We make an ansatz

$$
\chi_{b}(\lambda, \mu)=-\log \mu+\sum_{n=0}^{\infty} \mu^{-2 n} \chi_{b, n}(z)
$$

where $\chi_{b, n}(z)$ is a power series in $z=\lambda^{2} / \mu$, following [1], and obeys the boundary condition

$$
\begin{aligned}
& \chi_{b, 0}(0)=0 \\
& \chi_{b, n}(0)=f_{n}(1) .
\end{aligned}
$$

Through almost the same procedure, we obtain for the bosonic case,

$$
\begin{aligned}
& X_{b, 0}=-\log y \\
& X_{b, 1}=\frac{1}{12 y^{2}}
\end{aligned}
$$




$$
\begin{aligned}
& X_{b, 2}=\frac{1}{40 y^{4}}, \\
& X_{b, 3}=\frac{5}{252 y^{6}}, \\
& \text { etc. }
\end{aligned}
$$

Here $X_{b, n}(y)$ is defined as follows,

$$
\begin{aligned}
& X_{b, 0}(y)=-\log y+\chi_{b, 0}\left(y^{-1 /(2-R)}\right), \\
& X_{b, n}(y)=y^{-2 n} \chi_{b, n}\left(y^{-1 /(2-2 R)}\right) . \quad(n \geq 1)
\end{aligned}
$$

It is tempting to conjecture that

$$
X_{b, n}=\frac{f_{n}(1)}{y^{2 n}} \quad(n \geq 1),
$$

which implies that $\chi_{b}(\lambda, \mu)$ is independent of $\lambda$. In fact, it can be proved that this is the case, see appendix C

For type 0B case, the boundary condition is

$$
\chi_{B}(\lambda=0, \mu)=2 \chi_{b}(\lambda=0, \mu),
$$

but now $R=1 / 2$. That is,

$$
\chi_{B, 0}(0)=0, \quad \chi_{B, n \geq 1}(0)=f_{n}(1 / 2) .
$$

The form of $X_{B, n}$ is not $2 X_{b, n}$ since the equation is non-linear, and we obtain

$$
\begin{aligned}
& X_{B, 0}=-\log y \\
& X_{B, 1}=\frac{1}{12 y^{2}}+\frac{1}{8(y-1)^{2}}, \\
& X_{B, 2}=\frac{1}{40 y^{4}}+\frac{9}{64(y-1)^{4}}-\frac{9}{32(y-1)^{5}}, \\
& X_{B, 3}=\frac{5}{252 y^{6}}+\frac{45}{128(y-1)^{6}}+\frac{1215}{512(y-1)^{7}}+\frac{1323}{512(y-1)^{8}},
\end{aligned}
$$

It looks that the type $0 \mathrm{~B}$ result is simpler than the type $0 \mathrm{~A}$ result. Note that $X_{B, n}$ corresponds to $X_{ \pm, 2 n}$ in the type $0 \mathrm{~A}$ case. 


\section{Wilson line}

In [3], it is shown that the vev of Wilson line operator along the (Euclideanized) time direction provides the correct information on the target space geometry, assuming a holographic relation. It is interesting to check whether a similar calculation can be done in the cigar geometry in type 0 A string.

It is expected that the vev of the Wilson line is related to a holographically dual string theory by the following relation,

$$
\langle W\rangle=\left\langle e^{i \oint A}\right\rangle=\int D X e^{-S_{p}(X)},
$$

where $S_{p}(X)$ is the Polyakov action. There is another relation which uses the Nambu-Goto action,

$$
\left\langle\frac{1}{N} e^{i \oint A}\right\rangle=\int D X e^{-S_{N G}}
$$

as is used in [3]. These two relations would be different if the two string theories are different at the quantum level. At least we can say that the former has an advantage since the string coupling dependence is manifest. In the following, we will use the former.

The leading order behavior comes from the classical value of the action. Since the worldsheet of the string wraps all the spacetime, the worldsheet coordinates are identified with the spacetime ones, and the worldsheet metric is also the same as the spacetime one. Then,

$$
\begin{aligned}
S_{p} & =\frac{1}{2 \pi \alpha^{\prime}} \int d^{2} x \sqrt{g}+\Phi(0)-\frac{1}{4 \pi} \int d^{2} x R^{(2)} \log (\cosh r) \\
& =k\left(\Phi(0)-\Phi_{b}\right)+\Phi(0)+O(1),
\end{aligned}
$$

where $\Phi_{b}$ is the value of the dilaton at the point where the boundary theory lives. That is, the Wilson line behaves as

$$
\langle W\rangle \sim e^{-3 \Phi(0) / 2}
$$

Let us compare this result with a quantity derived from the matrix model. The vev of the Wilson line can be calculated as follows,

$$
\langle W\rangle_{m m}=\langle\operatorname{Tr} U\rangle \sim \partial_{\lambda} F\left(\lambda, \mu^{\prime}\right)
$$

Note that the vev of $\operatorname{Tr} V$ is the same since the perturbation is symmetric.

If we consider a limit $\lambda \rightarrow \infty$ while $\mu^{\prime}$ is fixed, in analogy with the bosonic case [3], we obtain

$$
\langle W\rangle_{m m} \sim \lambda \sim g_{s}^{-1}
$$

so this limit is not appropriate for the fermionic case. The correct behavior $\langle W\rangle_{m m} \sim g_{s}^{-3 / 2}$ of the Wilson line is obtained by taking another limit $\lambda, \mu^{\prime} \rightarrow \infty$ while $y$ is fixed. Therefore, the 
type 0A matrix model with the symmetric perturbation can be holographically dual to the type $0 \mathrm{~A}$ string on the Witten's black hole by taking the latter limit.

One interesting fact is that $\mathcal{N}=1 S L(2, R) / U(1)$ supercoset theory has an accidental $\mathcal{N}=2$ worldsheet supersymmetry [1]. Furthermore in [21], it is shown that the $\mathcal{N}=1$ supercoset theory is mirror to $\mathcal{N}=2$ Liouville theory. Thus from the holographic point of view it is natural that the limit of the deformed matrix model $\lambda, \mu^{\prime} \rightarrow \infty$ with $y$ fixed corresponds to the point of enhanced $\mathcal{N}=2$ worldsheet supersymmetry. This is an interesting matrix model realization of $\mathcal{N}=2$ Liouville theory and we should look for further evidences for this holographic relation. Note also that in terms of $\mathcal{N}=1$ superfield language, we are taking the limit where the coefficient of $\mathcal{N}=1$ Liouville potential terms are vanishing while keeping the coefficient of $\mathcal{N}=1$ sine-Liouville terms finite.

Note that we have considered the situation with $y=O(1)$. This seems to be justified by the singular behavior of the free energy at $y=1$. The $\log (1-z)$ term in the genus one free energy (5.30) would imply that a massless state appears when we take $y \rightarrow 1$ limit. This seems to be consistent with the fact that, in the worldsheet theory, there is an IR fixed point at which the supersymmetry is enhanced to $\mathcal{N}=2$. Note that at some general $R$, the structure of the tree level free energy is almost the same as that of the bosonic one except for a scaling of $R$, which would suggest that the type 0A matrix model with generic $R$ describes $\mathcal{N}=1$ Liouville theory with a perturbation as also claimed in [1]. According to this interpretation of the singularity at $y=1$, since this is an IR divergence, the singularity does not mean any inconsistency of the model, and therefore the above calculation of Wilson line around $y=1$ could be justified. Note that the higher order contribution to the Wilson line can be finite and non-zero by fine-tuning how fast $y$ goes to 1 , if the conjectured form (15.28) is correct. The above calculation suggests that in order to use the deformed matrix model to describe $\mathcal{N}=2$ Liouville theory properly, we should take account of additional degrees of freedom appearing in the limit of $y \rightarrow 1$. If we use the relation of the deformed matrix model to the topological string as explained in the next section, we can gain some insights for the additional degrees of freedom to be included.

\section{Relation to topological string}

The free energy of type $0 \mathrm{~A}$ matrix model is

$$
\partial_{\mu}^{3} F_{0 A}=R \operatorname{Im} \int_{0}^{\infty} d t e^{-i t(\mu-i f-i \epsilon)} \frac{t}{\sinh (t)} \frac{t / 2 R}{\sinh (t / 2 R)},
$$


while that of the bosonic string is

$$
\partial_{\mu}^{3} F_{b}=R \operatorname{Im} \int_{0}^{\infty} d t e^{-i t(\mu-i \epsilon)} \frac{t / 2}{\sinh (t / 2)} \frac{t / 2 R}{\sinh (t / 2 R)} .
$$

The bosonic case with $R=1$ produces the partition function of type IIB topological string on the conifold. The type $0 \mathrm{~A}$ case with $R=\frac{1}{2}$ and $f=0$ gives the twice of the partition function of the topological string on the conifold. Thus one might wonder if the theory is again related to a topological string. Indeed it is argued in 22] that corresponding topological string is defined on a $Z_{2}$ orbifold of the conifold. The $Z_{2}$ orbifold action on the conifold $x^{\prime} y^{\prime}=u v$ is given by

$$
\begin{aligned}
\left(x^{\prime}, y^{\prime}\right) & \rightarrow\left(-x^{\prime},-y^{\prime}\right) \\
(u, v) & \rightarrow(u, v) .
\end{aligned}
$$

If we choose invariant variables $x \equiv x^{\prime 2}, y \equiv y^{\prime 2}$, we have

$$
x y=(u v)^{2}
$$

The deformation is given by

$$
x y=(u v+\mu)^{2}-\frac{f^{2}}{2}
$$

where $f$ is related to the RR flux of type 0A model. It is explained in [22] that the geometry given by eq. (17.5) has two $S^{3}$ cycles, which are of size $\mu$ if $f=0$. Thus in the $\mu \rightarrow 0$ limit, two $S^{3}$ s are shrinking simultaneously. For $y=1$ case of the previous section, this is related to deforming the complex structures at infinities of the non-compact Calabi-Yau manifold with conifold-like singularities 9 . In our case, we have the same deformations for both winding modes and this should be related to the deformation of the curve $u v+\mu=0$. This would be quite close to the bosonic case and equivalent picture is also developed in [18. There the complex structure deformation is mapped to the deformation of the Fermi sea profile. It would be interesting to understand the integrable structures of the topological string on such $Z_{2}$ orbifold of the conifold related to the complex structure deformations at the asymptotic regions. 


\section{A Determinant representation}

The expression (4.1) can be written as follows,

$$
\begin{aligned}
& Z_{N}(\lambda, \tilde{\lambda})=\frac{1}{(N !)^{2}} \oint \prod_{i=1}^{N} \frac{d z_{i}}{2 \pi i} \frac{d \tilde{z}_{i}}{2 \pi i} \operatorname{det}_{i j} K\left(z_{i}, \tilde{z}_{j}\right) \operatorname{det}_{i j} \tilde{K}\left(\tilde{z}_{i}, z_{j}\right) \\
& =\frac{1}{(N !)^{2}} \oint \prod_{i=1}^{N} \frac{d z_{i}}{2 \pi i} \frac{d \tilde{z}_{i}}{2 \pi i} \sum_{\sigma \in S_{N}}(-1)^{\sigma} \prod_{k=1}^{N} K\left(z_{k}, \tilde{z}_{\sigma(k)}\right) \sum_{\sigma^{\prime} \in S_{N}}(-1)^{\sigma^{\prime}} \prod_{l=1}^{N} \tilde{K}\left(\tilde{z}_{l}, z_{\sigma^{\prime}(l)}\right) \\
& =\frac{1}{(N !)^{2}} \oint \prod_{i=1}^{N} \frac{d z_{i}}{2 \pi i} \frac{d \tilde{z}_{i}}{2 \pi i} \sum_{\sigma \in S_{N}}(-1)^{\sigma} \prod_{k=1}^{N} K\left(z_{k}, \tilde{z}_{\sigma(k)}\right) \sum_{\sigma^{\prime} \in S_{N}}(-1)^{\sigma^{\prime}} \prod_{l=1}^{N} \tilde{K}\left(\tilde{z}_{\sigma(l)}, z_{\sigma\left(\sigma^{\prime}(l)\right)}\right) \\
& =\frac{1}{(N !)^{2}} \oint \prod_{i=1}^{N} \frac{d z_{i}}{2 \pi i} \frac{d \tilde{z}_{i}}{2 \pi i} \sum_{\sigma \in S_{N}}(-1)^{\sigma} \prod_{k=1}^{N} K\left(z_{k}, \tilde{z}_{\sigma(k)}\right) \sum_{\tilde{\sigma} \in S_{N}}(-1)^{\tilde{\sigma}}(-1)^{\sigma} \prod_{l=1}^{N} \tilde{K}\left(\tilde{z}_{\sigma(l)}, z_{\tilde{\sigma}(l)}\right) \\
& =\frac{1}{(N !)^{2}} \oint \prod_{i=1}^{N} \frac{d z_{i}}{2 \pi i} \frac{d \tilde{z}_{i}}{2 \pi i} \sum_{\sigma \in S_{N}} \sum_{\tilde{\sigma} \in S_{N}}(-1)^{\tilde{\sigma}} \prod_{k=1}^{N} K\left(z_{k}, \tilde{z}_{\sigma(k)}\right) \tilde{K}\left(\tilde{z}_{\sigma(k)}, z_{\tilde{\sigma}(k)}\right) \\
& =\frac{1}{N !} \oint \prod_{i=1}^{N} \frac{d z_{i}}{2 \pi i} \sum_{\tilde{\sigma} \in S_{N}}(-1)^{\tilde{\sigma}} \prod_{k=1}^{N} \oint \frac{d \tilde{z}}{2 \pi i} K\left(z_{k}, \tilde{z}\right) \tilde{K}\left(\tilde{z}, z_{\tilde{\sigma}(k)}\right) \\
& =\frac{1}{N !} \oint \prod_{i=1}^{N} \frac{d z_{i}}{2 \pi i} \sum_{\tilde{\sigma} \in S_{N}}(-1)^{\tilde{\sigma}} \prod_{k=1}^{N} \mathcal{K}\left(z_{k}, z_{\tilde{\sigma}(k)}\right) \text {. }
\end{aligned}
$$

The summation over permutations can be rewritten as follows (see e.g. [23]),

$$
\begin{aligned}
& \frac{1}{N !} \oint \prod_{i=1}^{N} \frac{d z_{i}}{2 \pi i} \sum_{\sigma \in S_{N}}(-1)^{\sigma} \prod_{k=1}^{N} \mathcal{K}\left(z_{k}, z_{\sigma(k)}\right) \\
= & \frac{1}{N !} \sum_{\left\{d_{l}\right\}}^{\prime} \prod_{l=1}^{\infty}\left((-1)^{l+1}\right)^{d_{l}} \frac{N !}{\prod_{l=1}^{\infty}(l !)^{d_{l}} d_{l} !} \prod_{l=1}^{\infty}\left(\frac{l !}{l}\right)^{d_{l}} \prod_{l=1}^{\infty}\left(\operatorname{Tr} \mathcal{K}^{l}\right)^{d_{l}} \\
= & \sum_{\left\{d_{l}\right\}}^{\prime} \prod_{l=1}^{\infty} \frac{1}{d_{l} !}\left(\frac{(-1)^{l+1}}{l} \operatorname{Tr} \mathcal{K}^{l}\right)^{d_{l}},
\end{aligned}
$$

where $\sum^{\prime}$ represents the sum of $d_{l}$ with the constraint $\sum_{l=1}^{\infty} l d_{l}=N$. Then the grand canonical partition function is

$$
\begin{aligned}
Z(\lambda, \tilde{\lambda} ; \mu) & =\sum_{N=0}^{\infty} e^{2 \pi R \mu N} Z_{N}(\lambda, \tilde{\lambda}) \\
& =\sum_{\left\{d_{l}\right\}} \prod_{l=1}^{\infty} \frac{1}{d_{l} !}\left(\frac{(-1)^{l+1}}{l} e^{2 \pi R \mu l} \operatorname{Tr} \mathcal{K}^{l}\right)^{d_{l}} \\
& =\prod_{l=1}^{\infty} \exp \left[\frac{(-1)^{l+1}}{l} e^{2 \pi R \mu l} \operatorname{Tr} \mathcal{K}^{l}\right]
\end{aligned}
$$




$$
\begin{aligned}
& =\exp \left[\operatorname{Tr} \log \left(1+e^{2 \pi R \mu} \mathcal{K}\right)\right] \\
& =\operatorname{Det}\left(1+e^{2 \pi R \mu} \mathcal{K}\right) .
\end{aligned}
$$

\section{B Free field representation}

The partition function (3.11) can be rewritten in terms of the free boson CFT[19]. Define two free bosons

$$
\varphi_{\alpha}(z)=\hat{q}_{\alpha}+\hat{p}_{\alpha} \log z+\sum_{m \neq 0} \frac{H_{\alpha, n}}{n} z^{-n}
$$

where $\alpha=1,2$ and

$$
\left[\hat{p}_{\alpha}, \hat{q}_{\beta}\right]=\delta_{\alpha \beta}, \quad\left[H_{\alpha, m}, H_{\beta, n}\right]=m \delta_{\alpha \beta} \delta_{m+n, 0} .
$$

Two linear combinations $\phi(z), \tilde{\phi}(z)$ of $\varphi_{\alpha}(z)$, where

$$
\begin{aligned}
& \phi(z)=\varphi_{1}\left(z q^{1 / 2}\right)-\varphi_{2}\left(z q^{-1 / 2}\right) \\
& \tilde{\phi}(z)=\varphi_{1}\left(z q^{-1 / 2}\right)-\varphi_{2}\left(z q^{1 / 2}\right)
\end{aligned}
$$

are relevant for rewriting (3.11), and one can show that

$$
\begin{aligned}
& \left\langle l\left|e^{-\sum_{n=1}^{\infty}\left(t_{n} H_{n}+\tilde{t}_{n} \tilde{H}_{n}\right)}\left(\oint \frac{d z}{2 \pi i} \oint \frac{d \tilde{z}}{2 \pi i}: e^{\phi(z)}:: e^{-\tilde{\phi}(\tilde{z})}:\right)^{N} e^{\sum_{n=1}^{\infty}\left(t_{-n} H_{-n}+\tilde{t}_{-n} \tilde{H}_{-n}\right)}\right| l\right\rangle \\
= & (-1)^{N}(N !)^{2} q^{N\left(l_{1}+l_{2}\right)} f_{l}(t, \tilde{t}) Z_{N}(\lambda, \tilde{\lambda})
\end{aligned}
$$

where $l=l_{1}, l_{2}$ collectively denotes the eigenvalues of $\hat{p}_{\alpha}, f=l_{1}-l_{2}$ and

$$
f_{l}(t, \tilde{t})=\left\langle l\left|e^{-\sum_{n=1}^{\infty}\left(t_{n} H_{n}+\tilde{t}_{n} \tilde{H}_{n}\right)} e^{\sum_{n=1}^{\infty}\left(t_{-n} H_{-n}+\tilde{t}_{-n} \tilde{H}_{-n}\right)}\right| l\right\rangle .
$$

We have defined that

$$
H_{\alpha, n}|l\rangle=0 \quad(n>0), \quad \hat{p}_{\alpha}|l\rangle=l_{\alpha}|l\rangle,
$$

and $H_{n}, \tilde{H}_{n}$ are defined by the following expression of $\phi(z)$,

$$
\phi(z)=\hat{q}+\hat{p} \log z+\sum_{m \neq 0} \frac{H_{n}}{n} z^{-n},
$$

and similar for tilder.

Let us define the following quantity

$$
T_{l}(t, \tilde{t})=f_{l}(t, \tilde{t}) \sum_{N=0}^{\infty} e^{2 \pi R \mu N} q^{N\left(l_{1}+l_{2}\right)} Z_{N}(\lambda, \tilde{\lambda}) .
$$


By the momentum conservation, this can be written as

$$
T_{l}(t, \tilde{t})=\left\langle l\left|e^{-\sum_{n=1}^{\infty}\left(t_{n} H_{n}+\tilde{t}_{n} \tilde{H}_{n}\right)} g(\mu) e^{\sum_{n=1}^{\infty}\left(t_{-n} H_{-n}+\tilde{t}_{-n} \tilde{H}_{-n}\right)}\right| l\right\rangle,
$$

where

$$
g(\mu)=\exp \left(e^{\pi R \mu} \oint \frac{d z}{2 \pi i}\left(e^{\phi(z)}-e^{-\tilde{\phi}(z)}\right)\right) .
$$

There are two tensor operators

$$
\sum_{\alpha, \beta} m_{\alpha \beta} \oint \frac{d z}{2 \pi i} e^{\varphi_{\alpha}\left(z q^{1 / 2}\right)} \otimes e^{-\varphi_{\beta}\left(z q^{-1 / 2}\right)}, \quad m=\sigma^{1}, \sigma^{3},
$$

which commute with $g(\mu) \otimes g(\mu)$. This commuting property leads to the following identities

$$
\begin{aligned}
& \sum_{\alpha, \beta} m_{\alpha \beta} \oint \frac{d z}{2 \pi i}\left\langle l\left|e^{-\sum_{n=1}^{\infty}\left(t_{n} H_{n}+\tilde{t}_{n} \tilde{H}_{n}\right)} e^{\varphi_{\alpha}(z)} g(\mu) e^{\sum_{n=1}^{\infty}\left(t_{-n} H_{-n}+\tilde{t}_{-n} \tilde{H}_{-n}\right)}\right| k\right\rangle \\
& \times\left\langle l^{\prime}\left|e^{-\sum_{n=1}^{\infty}\left(t_{n}^{\prime} H_{n}+\tilde{t}_{n}^{\prime} \tilde{H}_{n}\right)} e^{-\varphi_{\beta}(z)} g(\mu) e^{\sum_{n=1}^{\infty}\left(t_{-n}^{\prime} H_{-n}+\tilde{t}_{-n}^{\prime} \tilde{H}_{-n}\right)}\right| k^{\prime}\right\rangle \\
= & \sum_{\alpha, \beta} m_{\alpha \beta} \oint \frac{d z}{2 \pi i}\left\langle l\left|e^{-\sum_{n=1}^{\infty}\left(t_{n} H_{n}+\tilde{t}_{n} \tilde{H}_{n}\right)} g(\mu) e^{\varphi_{\alpha}(z)} e^{\sum_{n=1}^{\infty}\left(t_{-n} H_{-n}+\tilde{t}_{-n} \tilde{H}_{-n}\right)}\right| k\right\rangle \\
& \times\left\langle l^{\prime}\left|e^{-\sum_{n=1}^{\infty}\left(t_{n}^{\prime} H_{n}+\tilde{t}_{n}^{\prime} \tilde{H}_{n}\right)} g(\mu) e^{-\varphi_{\beta}(z)} e^{\sum_{n=1}^{\infty}\left(t_{-n}^{\prime} H_{-n}+\tilde{t}_{-n}^{\prime} \tilde{H}_{-n}\right)}\right| k^{\prime}\right\rangle .
\end{aligned}
$$

These equations provide infinite number of differential equations which contain quantities

$$
T_{l k}(t, \tilde{t})=\left\langle l\left|e^{-\sum_{n=1}^{\infty}\left(t_{n} H_{n}+\tilde{t}_{n} \tilde{H}_{n}\right)} g(\mu) e^{\sum_{n=1}^{\infty}\left(t_{-n} H_{-n}+\tilde{t}_{-n} \tilde{H}_{-n}\right)}\right| k\right\rangle,
$$

and their derivatives. Since the operator $g(\mu)$ commutes with $\hat{p}_{1}+\hat{p}_{2}, T_{l k}(t, \tilde{t})$ vanish unless $l_{1}+l_{2}=k_{1}+k_{2}$, but since it does not commute with $\hat{p}_{1}-\hat{p}_{2}, T_{l k}(t, \tilde{t})$ is otherwise non-vanishing in general, and the system of equations is more complicated than that obtained from the Hirota bilinear equation for an ordinary integrable system.

One way to simplify the situation is to represent $T_{l k}(t, \tilde{t})$ in terms of $T_{l}(t, \tilde{t})$. Suppose that $l_{1}=k_{1}+m, l_{2}=k_{2}-m$ for a positive integer $m$. Then one can show that

$$
T_{l k}(t, \tilde{t})=\mathcal{I}_{m} \circ \mathcal{I}_{m-1} \circ \cdots \circ \mathcal{I}_{1}\left(T_{k}^{(m)}(t, \tilde{t})\right),
$$

where

$$
T_{k}^{(m)}(t, \tilde{t})=\left\langle l\left|e^{-\sum_{n=1}^{\infty}\left(t_{n} H_{n}+\tilde{t}_{n} \tilde{H}_{n}\right)}\left(\oint \frac{d z}{2 \pi i} e^{\phi(z)}\right)^{m} g(\mu) e^{\sum_{n=1}^{\infty}\left(t_{-n} H_{-n}+\tilde{t}_{-n} \tilde{H}_{-n}\right)}\right| k\right\rangle,
$$

and $\mathcal{I}_{m}$ is an integral transformation defined by

$$
\mathcal{I}_{m}(f(\mu))=2 \pi R e^{m \pi R \mu} \int_{\mu}^{\infty} d \mu^{\prime} e^{-\pi R(m+1) \mu^{\prime}} f\left(\mu^{\prime}\right) .
$$

$T_{k}^{(m)}(t, \tilde{t})$ can be written in terms of $T_{k}(t, \tilde{t})$ by moving all $e^{\phi(z)}$ to the left, and therefore we finally obtain a set of infinite number of integral-differential equations for $T_{k}(t, \tilde{t})$. 


\section{Proof of the $\lambda$-independence of the bosonic solution}

The second derivative of the free energy of the $\mathrm{c}=1$ bosonic string at the self-dual radius is

$$
\chi_{b}(\mu)=-\log \mu+\sum_{N=1}^{\infty} \frac{2 N-1}{2 N}\left|B_{2 N}\right| \mu^{-2 N} .
$$

To see that this is really a solution of the Toda equation (15.3), it is sufficient to see

$$
\left(\frac{\sin \left(\partial_{\mu} / 2\right)}{\partial_{\mu} / 2}\right)^{2} \chi_{b}(\mu)=-\log \mu .
$$

It is more convenient to consider

$$
\left(\frac{\sin \left(\partial_{\mu} / 2\right)}{\partial_{\mu} / 2}\right)^{2} \partial_{\mu} \chi_{b}(\mu)=-\frac{1}{\mu}
$$

and (C.2 follows from this.

One can show that

$$
\begin{aligned}
& \left(\frac{\sin \left(\partial_{\mu} / 2\right)}{\partial_{\mu} / 2}\right)^{2} \partial_{\mu} \chi_{b}(\mu) \\
= & -2 \sum_{K=0}^{\infty}(-1)^{K}(2 K) ! \mu^{-2 K-1} \sum_{k=0}^{K} \frac{|(2 k-1)| B_{2 k}}{(2 k) !(2 K-2 k+2) !} .
\end{aligned}
$$

To evaluate the sum, let us calculate a related sum

$$
f(x)=\sum_{K=0}^{\infty} \sum_{k=0}^{K} \frac{(2 k-1) B_{2 k}}{(2 k) !(2 K-2 k+2) !} x^{2 K},
$$

which is

$$
\begin{aligned}
f(x) & =\sum_{k=0}^{\infty} \frac{(2 k-1) B_{2 k}}{(2 k) !} x^{2 k} \sum_{l=0}^{\infty} \frac{x^{2 l}}{(2 l+2) !} \\
& =-\frac{x^{2} e^{x}}{\left(e^{x}-1\right)^{2}} \frac{\cosh x-1}{x^{2}} \\
& =-\frac{1}{2} .
\end{aligned}
$$

(C.3) follows from this result.

\section{Acknowledgments}

We thank Piljin Yi for the participation of our project at the initial stage and intensive discussions throughout the work. The work of J.P. was supported by Korea Science and Engineering Foundation (KOSEF) Grant R01-2004-000-10526-0 and by POSTECH BSRI research fund 1RB0410601. 


\section{References}

[1] V. Kazakov, I. Kostov and D. Kutasov, "A Matrix Model for the Two Dimensional Black Hole," Nucl. Phys. B622 (2002) 141, hep-th/0101011.

[2] E. Witten, "On string theory and black holes," Phys. Rev, D44 (1991) 314.

[3] T. Suyama and P. Yi, 'A holographic view on matrix model on black hole," JHEP 0402:017 (2004), hep-th/0401078

[4] N. Berkovits, S. Gukov, B.C. Vallilo, "Superstrings in 2D Backgrounds with R-R Flux and New Extremal Black Holes," Nucl. Phys. B614 (2001) 195, hep-th/0107140.

[5] S. Gukov, T. Takayanagi, and N. Toumbas, "Flux backgrounds in 2D string theory," JHEP 0403:017 (2004), hep-th/0312208.

[6] D. Ghoshal and C. Vafa, "c=1 string as the topological theory of the conifold," Nucl. Phys. B453 (1995) 121, hep-th/9506122.

[7] A. Strominger, "Massless black holes and conifolds in string theory," Nucl. Phys. B451 (1995) 96, hep-th/9504090.

[8] H. Ooguri and C. Vafa, "Two-dimensional black hole and singularities of CY manifolds," Nucl. Phys. B463 (1996) 55, hep-th/9511164.

[9] M. Aganagic, R. Dijkgraaf, A. Klemm, M. Marino and C. Vafa, "Topological strings and integrable hierarchies," hep-th/0312085.

[10] U. Danielsson, M. Olsson and M. Vonk, "Matrix models, 4D black holes and topological strings on noncompact Calabi-Yau manifolds," JHEP 0411 (2004) 007, hep-th/0410141. U. Danielsson, J. Gregory, M. Olsson, P. Rajan and M. Vonk, "Type 0A 2-D blackhole thermodynamics and their deformed matrix model," JHEP 0404 (2004) 065, hep-th/0402192.

[11] R. Dijkgraaf, H. Verlinde, E. Verlinde, "String propagation in a black hole geometry," Nucl. Phys. B371 (1992) 269.

[12] A.A. Tseytlin, "Effective action of gauged WZW model and exact string solutions," Nucl. Phys. B399 (1993) 601.

[13] V. Fateev, A. Zamolodchikov and Al. Zamolodchikov, unpublished.

[14] I. Jack, "Exact bosonic and supersymmetric string black hole solutions," Nucl. Phys B393 (1993) 95, hep-th/9201039. 
[15] M. Douglas, I. Klebanov, D. Kutasov, J. Maldacena, E. Martinec and N. Seiberg, "A new hat for the c=1 matrix model," hep-th/0307195.

[16] T. Takayanagi, N. Toumbas, "A Matrix Model Dual of Type 0B String Theory in Two Dimensions," JHEP 0307 (2003) 064, hep-th/0307083.

[17] J. McGreevy and H. Verlinde, "Strings from tachyons: the c=1 matrix reloaded," JHEP 0312:054 (2003), hep-th/0304224.

[18] S. Alexandrov, V. Kazakov and I. Kostov, "Time dependent background of 2-D string theory," Nucl.Phys. B640 (2002) 119.

[19] X. Yin, "Matrix models, integrable structure and T-duality of Type 0 string theory," hep-th/0312236

[20] S. Alexandrov, V. Kazakov and D. Kutasov, "Nonperturbative effects in matrix models and D-branes," JHEP0309:057 (2003), hep-th/0306177.

[21] K. Hori and A. Kapustin, "Duality of the fermionic 2-D black hole and $\mathcal{N}=2$ Liouville theory as mirror symmetry," JHEP 0101:045 (2001), hep-th/0104202

[22] H. Ita, H. Nieder, Y. Oz and T. Sakai, "Topological B model, matrix models, $\hat{c}=1$ strings and quiver gauge theories," JHEP 0405:058 (2004), hep-th/0403256

[23] V.A. Kazakov, I.K. Kostov, N. Nekrasov, "D-particles, Matrix Integrals and KP hierarchy," Nucl. Phys. B557 (1999) 413, hep-th/9810035. 